\title{
Nonsy Load Flow: Smart Grid Load Flow Using Non-Synchronized Measurements
}

\author{
Alireza Bahmanyar ${ }^{*}$, Abouzar Estebsari $^{\dagger}$, Amin Bahmanyar ${ }^{\ddagger}$, Ettore Bompard ${ }^{\dagger}$ \\ *Department of Electrical Engineering, Iran University of Science and Technology, Tehran, Iran, 1684613114 \\ ${ }^{\dagger}$ Department of Energy, Politecnico di Torino, Turin, Italy, 10129 \\ ${ }^{\ddagger}$ Department of Electrical Engineering, Bojnourd Branch, Islamic Azad University, Bojnourd, Iran, 9417697796
}

\begin{abstract}
This paper presents a novel algorithm for load flow analysis in smart grids, using non-synchronized measurements collected at the main substation and at the terminals of Distributed Generators (DGs) and microgrids. This allows the use of already available measurements along with a proper communication system to calculate the magnitude and phase angle of nodal voltages, power flow in each branch, power injected by each electricity source, and system losses. The proposed non-synchronized measurements-based load flow (Nonsy load flow) algorithm is based on the conventional backward-forward sweep and it considers the synchronization angles as unknown variables to be calculated. Simulation studies on a smart grid model with several DG units and microgrids validate the performance of the proposed method. In all the studied cases, the load flow results are accurate and the unknown synchronization angles are precisely calculated as a byproduct of the algorithm without any significant extra computational effort. The calculated synchronization angles can satisfy the need of other smart grid applications requiring synchronized measurements.
\end{abstract}

Keywords- distributed generation; load flow; smart grid; synchronization, smart meters.

\section{INTRODUCTION}

Load flow is the most basic tool for power systems analysis and the basis of many other studies such as planning and optimization. Unlike their transmission counterparts, distribution networks are usually tree like radial networks originating from a substation and delivering the electricity to final customers in lower voltage levels through their many branches and tapped laterals. The well-known transmission load flow algorithms such as Gauss-Seidal, Newton-Raphson and its decoupled versions are seldom applicable to distribution level, due to their following special features:

- High R/X ratios;

- Radial or weakly meshed structure;

- Multi phase unbalanced lines;

- Unbalanced and distributed loads.

The proposed load flow methods for distribution networks can be classified to phase frame and sequence frame load flow approaches. Phase frame approaches are carried out in three phases a-b-c, and can be classified to forward and backward sweep methods, which are based on current summation, power summation or admittance summation, compensation-based methods, implicit $\mathrm{Z}$ bus methods, modified newton or newton like methods and other power flow methods. The sequence frame power flow approaches use decoupled positive, negative and zero sequence networks of unbalanced three-phase system to solve the unbalanced three-phase load flow [1].

Today, the growing electricity demand along with the need to counteract the global warming is driving the evolution of smart grids. There are some proven technologies, which will certainly have a role in distribution systems evolution. This includes advanced digital meters, distribution automation, lowcost communication systems, and distributed energy resources [2]. A smart grid accommodates a wide variety of generation options changing the traditional radial distribution to networks with non-radial power flows. Therefore, traditional distribution analysis methods such as radial load flow algorithms will not be applicable to such networks. However, load flow calculations are yet required to assess voltage profile, power flows and losses, to determine the basic capacity and voltage regulation issues associated with Distributed Generation (DG) interconnection, as well as to support subsequent analyses, such as reliability and power quality [3].

Several methods are developed for load flow calculations in distribution networks with DG. The method proposed in [4] models the synchronous or induction generator-based DGs by their Thevenin or Norton equivalents. Many other papers model the DGs as PQ or PV nodes to consider their penetration in distribution systems. For example, the method proposed in [5] represents all DGs as PV nodes, which inject reactive power to maintain a specified voltage value. To take into account the reactive power limits of DGs, the calculated DG reactive power is compared with its upper or lower limits and if it violates the limits, the model switches from PV to PQ mode. In [6] authors model small DG units as PQ nodes and large ones as PV nodes. A suitable model for each DG unit requires knowledge of the DG operation and the type of its connection to the grid (i.e. direct or indirect). In [7] authors investigate different types of DGs and derive a PV or PQ model for each type of them. Also in [8], the authors propose a constant power factor, a constant voltage or a variable reactive power model to integrate different types of DGs into load flow calculations. Although some of these methods have shown to have acceptable results, they are more appropriate for distribution network planning studies. In distribution system operational analysis, such as fault location studies, such simplifications in modelling may not provide the desired accuracy. On the other

This work was partially supported by FLEXMETER, which is an EU Horizon 2020 project under grant agreement no. 646568 . 
hand, detailed models of DGs complicate the calculations and significantly increases the computational time.

The metering infrastructure of distribution systems has been the focus of most recent investigations and relevant investments. FLEXMETER project, as an example, is one of the leading EU Horizon 2020 projects aiming at developing and demonstrating a flexible smart metering architecture for different general-purpose services [9]. Having the measurements deployed at DG terminals along with a proper communication system provides new possibilities for distribution analysis methods.

The employed DG technologies (i.e. synchronous generator, asynchronous generator, inverter-based) and the different ways for interface (i.e. direct, indirect) determine the fault current contributions of DGs and microgrids. Yet, if DGs and microgrids current phasors are available, during load flow calculations, each DG unit or microgrid participation can be simply considered as a current injection and no information regarding their parameters and interfaces will be required. This approach can provide very accurate results, but it demands a precise measurement synchronization infrastructure. Synchronization can be achieved using the Global Positioning System (GPS) or a computer network. However, even in the recent smart metering projects, the measurements are mostly not synchronized, mainly due to economic issues. This fact limits the practical application of current injection model of DGs, despite its accuracy.

In this paper, we present a novel load flow algorithm, using non-synchronized measurements collected at the main substation and at the terminals of DGs and microgrids. This allows the use of already available measurements to perform the load flow analysis. The proposed algorithm is based on the conventional backward-forward sweep load flow and it considers the synchronization angles as unknown variables to be calculated. Simulation studies on a smart grid model with several DG units and microgrids validates the good performance of the proposed method. The unknown synchronization angles are determined precisely which can be employed for other applications such as smart grid monitoring or fault location [10].

\section{NON-SYNCHRONIZED MEASUREMENTS-BASED LOAD FLOW (NONSY LOAD FLOW)}

A sinusoidal waveform whose amplitude, angular frequency, and initial phase are time-invariant, can be represented by a unique rotating complex number known as a phasor. Consider the simple system shown in Fig. 1, which carries electricity from the main substation and distributed energy resources to distribution transformers located near customer's premises. The sinusoidal waveform of the main component of the main substation voltage can be written as follows:

$$
v_{S}(t)=V_{S}^{m} \cos (w t+\alpha)
$$

As illustrated in Fig. 2, the phasor representation of this sinusoid is given by:

$$
V_{s}=\left(V_{s}^{m} / \sqrt{2}\right) e^{j \alpha}=\left(V_{s}^{m} / \sqrt{2}\right)(\cos \alpha+j \sin \alpha)
$$

where $V s^{m}$ and $\alpha$ are the magnitude and angular displacement of the substation voltage. If we take the main substation voltage phasor as the phasor reference $(\alpha=0)$, the current $\left(i_{x}\right)$ and voltage $\left(v_{x}\right)$ waveforms of each DG unit or microgrid can be written as follows:

$$
\begin{aligned}
& v_{x}(t)=V_{x}^{m} \cos \left(w t-\theta_{x}\right) \\
& i_{x}(t)=I_{x}^{m} \cos \left(w t-\theta_{x}-\varphi_{x}\right)
\end{aligned}
$$

where, as shown in the phasor representation of Fig. 3, $\theta_{x}$ and $\varphi_{x}$ are, respectively, the angle between the main substation voltage $\left(V_{S}\right)$ and $V_{x}$ and the angle between $V_{x}$ and $I_{x}$.

When all the measurements at DGs and microgrids terminals are synchronized by the main substation voltage, either internally or using GPS, $\theta_{x}, \varphi_{x}, V_{x}^{m}$ and $I_{x}^{m}$ are all known values. Therefore, during load flow, participation of each DG unit or microgrid can be simply considered as a current injection (a negative constant current load). Although this method is simple and it does not require any information regarding the microgrid or DG parameters or their interface model, it demands a precise measurement synchronization infrastructure. This fact limits the practical application of current injection model of DGs.

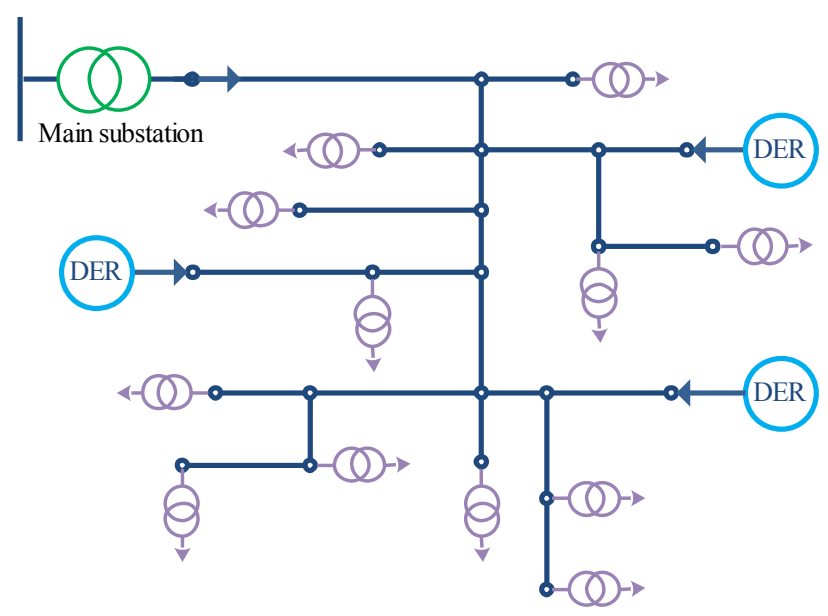

Fig. 1. Simple distribution network with multiple distributed energy resources

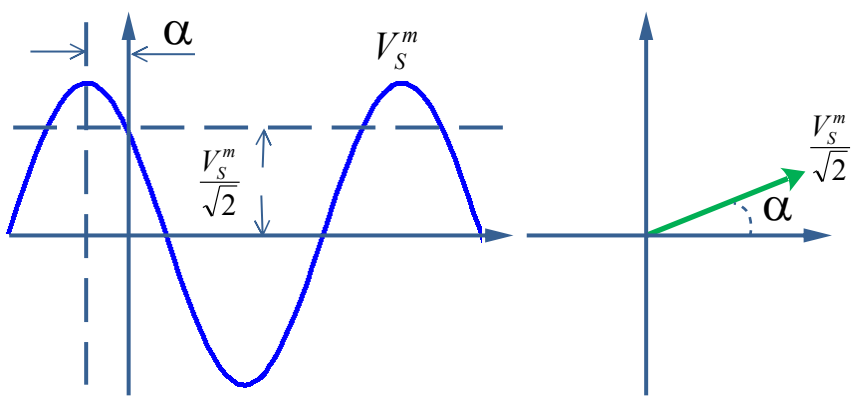

Fig. 2. Waveform and phasor representation of the substation voltage 


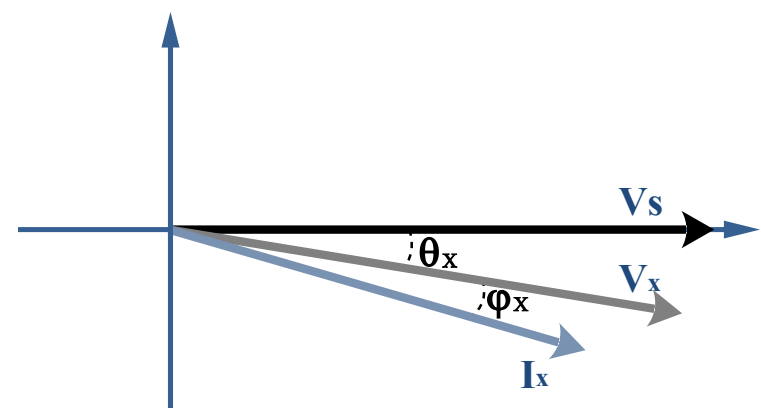

Fig. 3. Phasor representation of the current and voltage of unit $x$, taking the main substation voltage as reference.

When the meters are not synchronized, they still can provide the voltage and current magnitudes and their angular difference $\left(\varphi_{x}\right)$, but the synchronization angle $\left(\theta_{x}\right)$ is an unknown variable.

In the proposed load flow algorithm, the synchronization angles are initially assumed to be zero $\left(\theta_{x}^{(0)}=0\right)$. Therefore, having the values of the other variables $\left(\varphi_{x}, V_{x}^{m}\right.$ and $\left.I_{x}^{m}\right)$, the load flow algorithm can consider each DG unit or microgrid participation as a current injection.

$$
I_{x}^{(k)}=I_{x}^{m} \angle\left(-\varphi_{x}-\theta_{x}^{(k-1)}\right)
$$

where $k$ denotes the iteration.

Similar to traditional backward-forward sweep load flow algorithm, in each iteration, Nonsy load flow performs a backward sweep to calculate the line currents and a forward sweep to update the nodal voltages.

At the end of each backward/forward cycle, the algorithm uses the angle of the calculated DG and microgrid terminal voltages (i.e. $\theta_{x}$ ) to modify their current phasors, using (4). Indeed, the Nonsy load flow considers the synchronization angles as unknown variables to be calculated and repeats the backward/forward and current phasors modification cycle until the convergence is achieved:

$$
\max \left\{\left|V^{(k)}-V^{(k-1)}\right|\right\}<\varepsilon
$$

where $V^{(k)}$ is the vector of calculated voltages for all nodes at the $k^{\text {th }}$ iteration.

Fig. 4 presents the flowchart of the Nonsy load flow algorithm.

\section{CASE STUDY AND RESULTS}

Simulation tests are performed on a 98-node real-life distribution feeder, shown in Fig. 5. The test system is simulated in MATLAB/Simulink. To form a smart grid, the modeled grid is modified by adding an average model of a 1.5 MW doubly fed induction generator (DFIG) along with its wind turbine, three similar microgrids and two similar average models of a $400 \mathrm{~kW}$ PV farm. Microgrids are modeled in detail as proposed in [11]. Voltage and current waveforms at each source terminal are recorded with a sampling rate of 256 samples per cycle and the full cycle discrete Fourier transform is employed to calculate the fundamental voltage and current phasors.

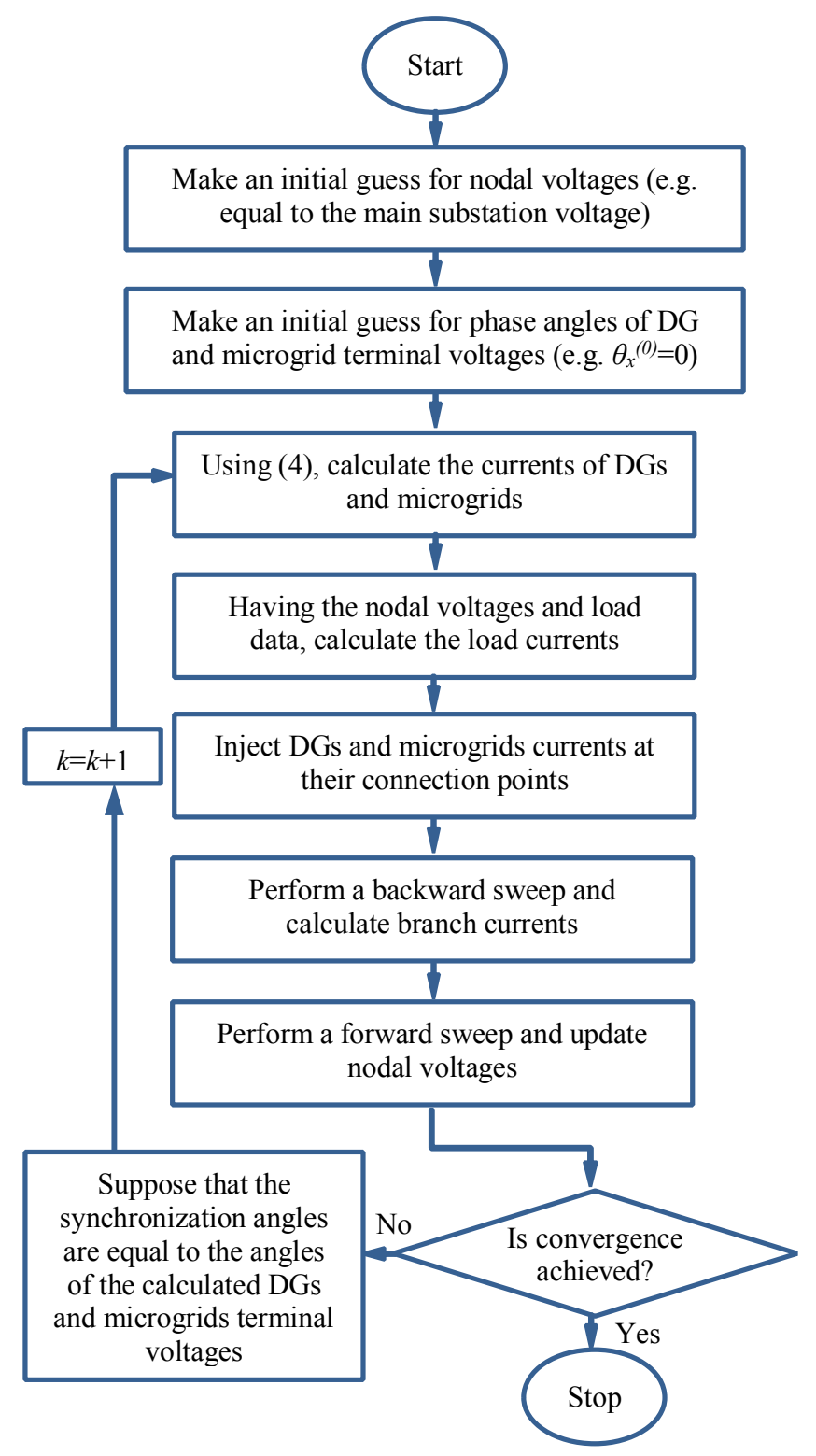

Fig. 4. Flowchart of the proposed Nonsy load flow algorithm

The Nonsy load flow algorithm uses the magnitude and angular difference of non-synchronized voltages and currents recorded at all source terminals to calculate the magnitude and phase angle of nodal voltages, power flow in each branch, power injected by each source and system losses.

The proposed algorithm is tested under different loading conditions. For all studied cases, the same initial assumptions are made for nodal voltages and branch currents (i.e. equal to the values measured at the main substation). The algorithm is not sensitive to initial guess and on a personal computer with 2-GHz Intel Core 2 Duo processor and 2 GB of RAM, it took about $0.15 \mathrm{~s}$ and less than 6 iterations to converge. No 
divergence is observed under the studied scenarios, however under very large measurements error, the algorithm may fail to converge.

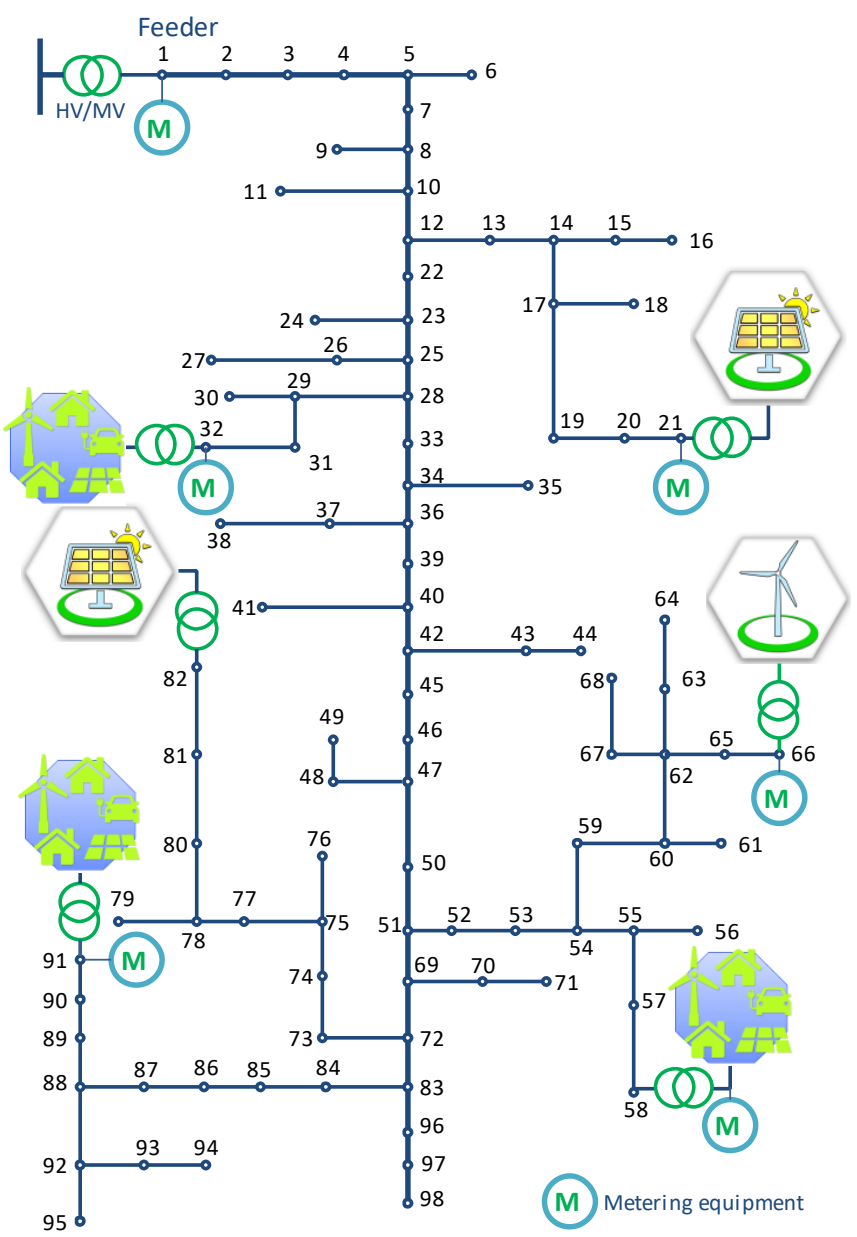

Fig. 5. The 98-node smart distribution test system

Fig. 6a shows the actual values of nodal voltages generated during the simulation with respect to the voltages calculated using the Nonsy load flow under heavy load condition.

The calculated values perfectly match the actual values, and as shown in Fig. 6b, for all nodes the difference is less than 0.001 p.u. In Table. I, the actual and calculated nodal voltages of some of the test network nodes are presented and compared.

Fig. 7 shows the difference between the actual and calculated phase angles of nodal voltages. The differences are very small and are mainly due to quantization errors in phasor estimation.

The proposed algorithm not only provides accurate results without synchronized measurements, but it is also able to estimate the synchronization angles. As shown in Fig. 8, Nonsy load flow provides a precise estimation of synchronization angles that can satisfy the requirement of other smart grid applications relying on synchronized measurements. Similar results are obtained for light load condition.

(a)
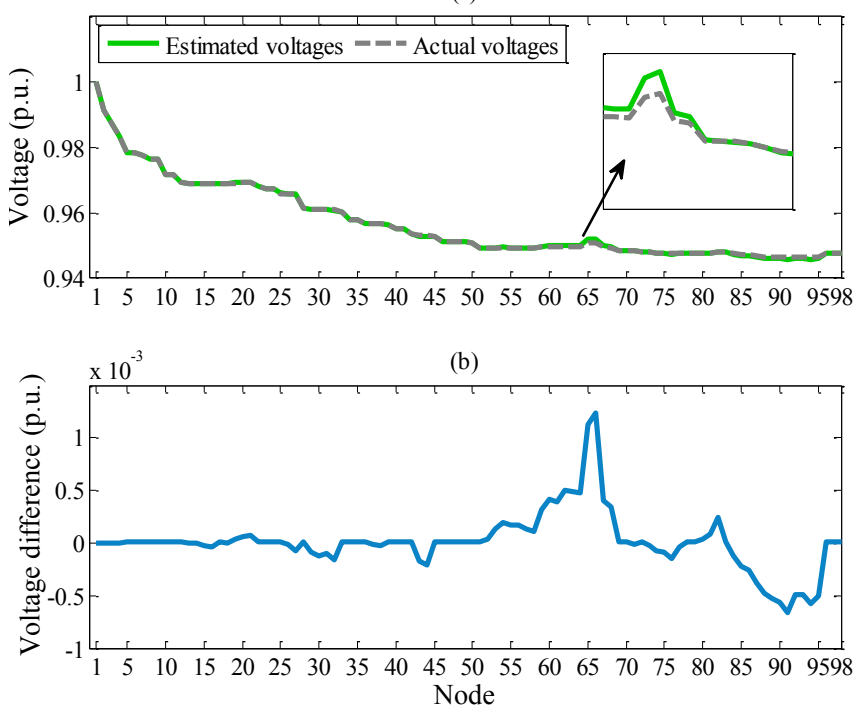

Fig. 6. (a). Actual and estimated values of nodal voltages. (b). Difference between the actual and estimated voltages

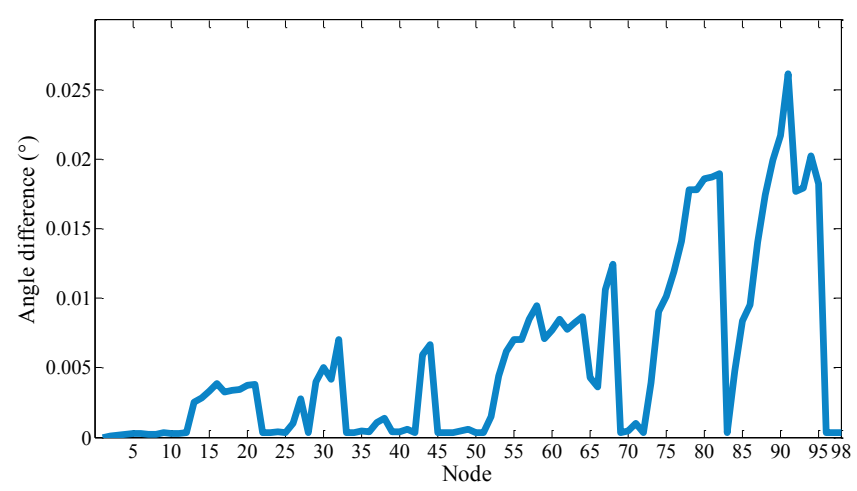

Fig. 7. Estimated and actual values of synchronization angles

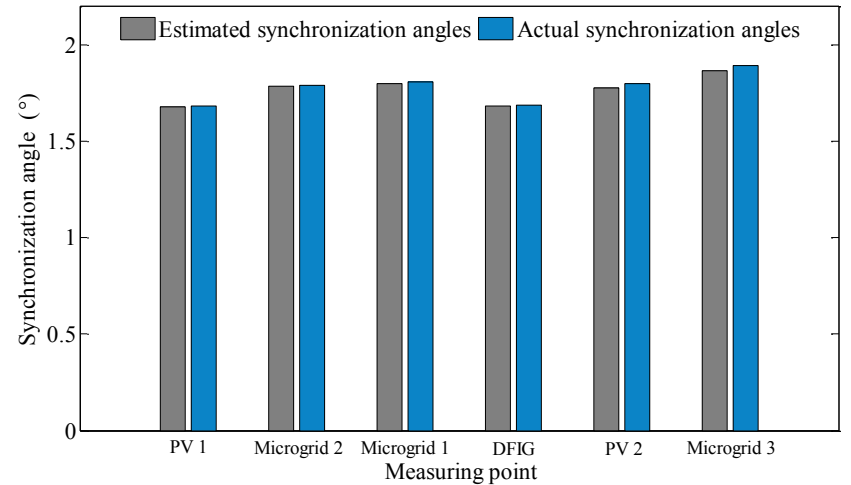

Fig. 8. Estimated and actual values of synchronization angles 
TABLE I. COMPARISON OF THE ACTUAL AND CALCULATED VOLTAGES

\begin{tabular}{|c|c|c|c|}
\hline Node No. & $\begin{array}{c}\text { Actual V } \\
\text { (pu) }\end{array}$ & $\begin{array}{c}\text { Calculated } \\
\text { V (pu) }\end{array}$ & Error $\times 1000$ \\
\hline 1 & 1 & 1 & 0 \\
\hline 4 & 0.9834 & 0.9834 & 0.00463 \\
\hline 7 & 0.9777 & 0.9778 & 0.0083 \\
\hline 10 & 0.9717 & 0.9717 & 0.01002 \\
\hline 13 & 0.9691 & 0.9691 & 0.00103 \\
\hline 16 & 0.969 & 0.969 & 0.03885 \\
\hline 19 & 0.9692 & 0.9692 & 0.03212 \\
\hline 22 & 0.9681 & 0.9681 & 0.01098 \\
\hline 25 & 0.966 & 0.966 & 0.01145 \\
\hline 28 & 0.9615 & 0.9615 & 0.01249 \\
\hline 31 & 0.9612 & 0.9611 & 0.09255 \\
\hline 34 & 0.958 & 0.958 & 0.01327 \\
\hline 37 & 0.9569 & 0.9568 & 0.01124 \\
\hline 40 & 0.9552 & 0.9553 & 0.01375 \\
\hline 43 & 0.9532 & 0.953 & 0.17722 \\
\hline 46 & 0.9514 & 0.9514 & 0.01428 \\
\hline 49 & 0.9513 & 0.9513 & 0.00705 \\
\hline 52 & 0.9492 & 0.9493 & 0.03989 \\
\hline 55 & 0.9492 & 0.9494 & 0.17566 \\
\hline 58 & 0.9491 & 0.9492 & 0.11083 \\
\hline 61 & 0.9495 & 0.9499 & 0.38774 \\
\hline 64 & 0.9496 & 0.9501 & 0.4727 \\
\hline 67 & 0.9495 & 0.9499 & 0.40493 \\
\hline 70 & 0.9483 & 0.9483 & 0.01113 \\
\hline 73 & 0.948 & 0.948 & 0.0211 \\
\hline 76 & 0.9475 & 0.9474 & 0.14194 \\
\hline 79 & 0.9476 & 0.9476 & 0.0056 \\
\hline 82 & 0.9479 & 0.9482 & 0.25077 \\
\hline 85 & 0.9473 & 0.9471 & 0.22364 \\
\hline 88 & 0.9466 & 0.9461 & 0.47926 \\
\hline 91 & 0.9464 & 0.9458 & 0.66399 \\
\hline 94 & 0.9464 & 0.9458 & 0.57485 \\
\hline 97 & 0.9478 & 0.9478 & 0.01512 \\
\hline
\end{tabular}

\section{CONCLUTions}

A new method for load flow calculation in smart grids is proposed in this paper. The method uses the non-synchronized measurements collected at the main substation and at the terminals of DGs and microgrids. The major contributions of the proposed load flow algorithm can be summarized as follows:
- The proposed method is algorithmically simple.

- It allows the use of comparatively cheaper nonsynchronized measurements to perform load flow analysis.

- It does not require any information about the several DGs and microgrids connected to the grid regarding their parameters and interface type.

- Simulation results considering the detailed model of different types of DGs and microgrids validates the high accuracy of the proposed method.

- The method provides a precise estimation of synchronization angles and can be of great help to distribution system operators satisfying the requirement of other smart grid applications relying on synchronized measurements.

\section{REFERENCES}

[1] K. Balamurugan and D. Srinivasan, "Review of power flow studies on distribution network with distributed generation," in IEEE Ninth International Conference on Power Electronics and Drive Systems (PEDS), 2011, pp. 411-417.

[2] R. E. Brown, "Impact of smart grid on distribution system design," in IEEE Power and Energy Society General Meeting-Conversion and Delivery of Electrical Energy in the 21st Century, 2008, pp. 1-4.

[3] J. A. Martinez and J. Mahseredjian, "Load flow calculations in distribution systems with distributed resources. A review," in IEEE Power and Energy Society General Meeting, 2011, pp. 1-8.

[4] T.-H. Chen, M.-S. Chen, T. Inoue, P. Kotas, and E. A. Chebli, "Threephase cogenerator and transformer models for distribution system analysis," IEEE Transactions on Power Delivery, vol. 6, pp. 1671-1681, 1991.

[5] H. E. Farag, E. El-Saadany, R. El Shatshat, and A. Zidan, "A generalized power flow analysis for distribution systems with high penetration of distributed generation," Electric Power Systems Research, vol. 81, pp. 1499-1506, 2011.

[6] S. Khushalani, J. M. Solanki, and N. N. Schulz, "Development of threephase unbalanced power flow using PV and PQ models for distributed generation and study of the impact of DG models," IEEE Transactions on Power Systems, vol. 22, pp. 1019-1025, 2007.

[7] S. Moghaddas-Tafreshi and E. Mashhour, "Distributed generation modeling for power flow studies and a three-phase unbalanced power flow solution for radial distribution systems considering distributed generation," Electric Power Systems Research, vol. 79, pp. 680-686, 2009.

[8] J.-H. Teng, "Modelling distributed generations in three-phase distribution load flow," IET generation, transmission \& distribution, vol. 2, pp. 330-340, 2008.

[9] A. Bahmanyar, A. Estebsari, E. Pons, E. Patti, S. Jamali, E. Bompard, et al., "Emerging smart meters in electrical distribution systems: Opportunities and challenges," in 24th Iranian Conference on Electrical Engineering (ICEE), 2016, pp. 1082-1087.

[10] A. Bahmanyar, S. Jamali, A. Estebsari, and E. Bompard, "A comparison framework for distribution system outage and fault location methods," Electric Power Systems Research, vol. 145, pp. 19-34, 2017.

[11] S. Papathanassiou, N. Hatziargyriou and K. Strunz, "A benchmark low voltage microgrid network," Proceedings of the CIGRE symposium: power systems with dispersed generation, 2005, pp. 1-8. 